\title{
Des allégories des Saisons sur les tissus coptes
}

Allegories of the Seasons on Coptic textiles

\section{Amandine Mérat}

\section{OpenEdition}

Journals

Édition électronique

URL : http://journals.openedition.org/cel/525

DOI : $10.4000 /$ cel.525

ISSN : 2262-208X

Éditeur

École du Louvre

Édition imprimée

Date de publication : 1 mars 2013

Référence électronique

Amandine Mérat, "Des allégories des Saisons sur les tissus coptes », Les Cahiers de l'École du Louvre [En ligne], 2 | 2013, mis en ligne le 01 mars 2013, consulté le 03 mai 2019. URL : http:// journals.openedition.org/cel/525; DOI : 10.4000/cel.525

\section{(c) $(1) \&$}

Les Cahiers de l'École du Louvre sont mis à disposition selon les termes de la licence Creative Commons Attribution - Pas d'Utilisation Commerciale - Pas de Modification 4.0 International. 


\section{Des allégories des Saisons sur les tissus coptes}

\section{Amandine Mérat}

Parmi les milliers de textiles ornés en tapisserie retrouvés en Égypte et remontant à la période byzantine (fin du IV siècle-VII siècle après J.-C.) figurent de nombreux textiles décorés de visages ou de bustes, dont l'identification se révèle souvent délicate. La plupart est auréolé d'un nimbe, aussi les chercheurs ont-ils fréquemment été tentés d'y voir la représentation de saints. Parfois, l'identification même fait défaut, l'auteur préférant simplement parler de «buste», de "portrait» ou de "personnage », souvent sans avancer d'hypothèse plus précise ${ }^{1}$. Plusieurs textiles particulièrement représentatifs de ce cas de figure et appartenant à diverses collections - musée du Louvre, de Rennes et de Rouen - ont fait l'objet d'une étude iconographique approfondie ${ }^{2}$, ayant abouti à de nouvelles propositions d'identification, dont nous présentons ici les résultats.

\section{Des personnages énigmatiques}

Le premier de ces textiles ${ }^{3}$, découvert par Albert Gayet à Antinoé4, présente sur un fond de toile de lin écru plusieurs éléments en tapisserie de lin et laine. Malgré les lacunes, la répartition du décor reste très lisible : des motifs de fleurs répartis régulièrement forment un cadre carré dont seuls restent trois côtés. Aux deux angles conservés s'épanouissent de grosses fleurs à cœur jaune cerné de noir, qu'encadrent deux corolles de pétales blanc, rose et rouge pour la première, jaune cerné de vert pour la seconde. Entre ces éléments, sur chacun des côtés du carré sont disposés en ligne trois motifs floraux plus petits. Deux fleurs à tige et feuilles bleues, cœur jaune et trois pétales blanc, rose et rouge, encadrent une autre fleur, à quatre pétales et feuilles vertes. Au centre du carré ainsi formé se trouve un médaillon à fond rouge où prend place une figure féminine, le corps coupé en dessous des hanches (fig. 1). Traité de façon très géométrique, le visage encadré de cheveux noirs se détache sur un nimbe bleu cerné de blanc et de noir. La bouche est dessinée par deux lignes de laine noire, les yeux sont des carrés avec une pupille centrale, le nez et les sourcils sont marqués par des traits disposés perpendiculairement. Le corps dénudé porte une ceinture et un drapé vert et ocre flottant dans son dos. La femme est parée de bracelets et d'un collier blanc et rouge. Elle tient dans la main gauche une corbeille chargée de fruits, d'où s'échappe un élément végétal (épi de blé ?) et, dans la main droite, un objet recourbé (faucille ?), au dessus duquel prend place une étoile cruciforme.

1. Voir par exemple Yvonne Bourgon-Amir, « Section II : Les figures seules », Les Tapisseries coptes du Musée historique des Tissus de Lyon, Montpellier, Publications de la rechercheUniversité de Montpellier, 1993, pp. 98-121.

2. Amandine Mérat, «Les allégories de la Terre et des Saisons. Des sources littéraires et iconographiques gréco-romaines aux textiles byzantins », mémoire de recherche de l'École du Louvre, Roberta Cortopassi (dir.), septembre 2010. Nous exprimons à Mme Cortopassi, conservateur au Centre de Recherche et de Restauration des Musées de France - Filière Arts Décoratifs, nos remerciements les plus chaleureux pour l'aide apportée à la rédaction de cet article.

3. Inv. Musée du Louvre E 29288. Nous tenons à remercier ici Mmes MarieHélène Rutchowscaya et Dominique Benazeth, conservateurs à la section copte du département des Antiquités égyptiennes du musée du Louvre, de nous avoir permis d'étudier au sein des réserves les deux textiles présentés.

4. Ville de Moyenne Égypte fondée en 130 après J.-C. par Hadrien en I'honneur de son favori Antinoüs, mort noyé dans le Nil. Envoyé par l'industriel lyonnais Émile Guimet, Albert Gayet (1856-1916), élève de Gaston Maspero, y mena des fouilles entre 1895 et 1911, dont les découvertes, après présentation au musée Guimet de Lyon, étaient dispersées dans les musées français et étrangers. Pour une étude complète de Gayet et Antinoé, voir Florence Calament, La Révélation d'Antinoé par Albert Gayet. Histoire, archéologie, muséographie, Le Caire, IFAO, 2005. 


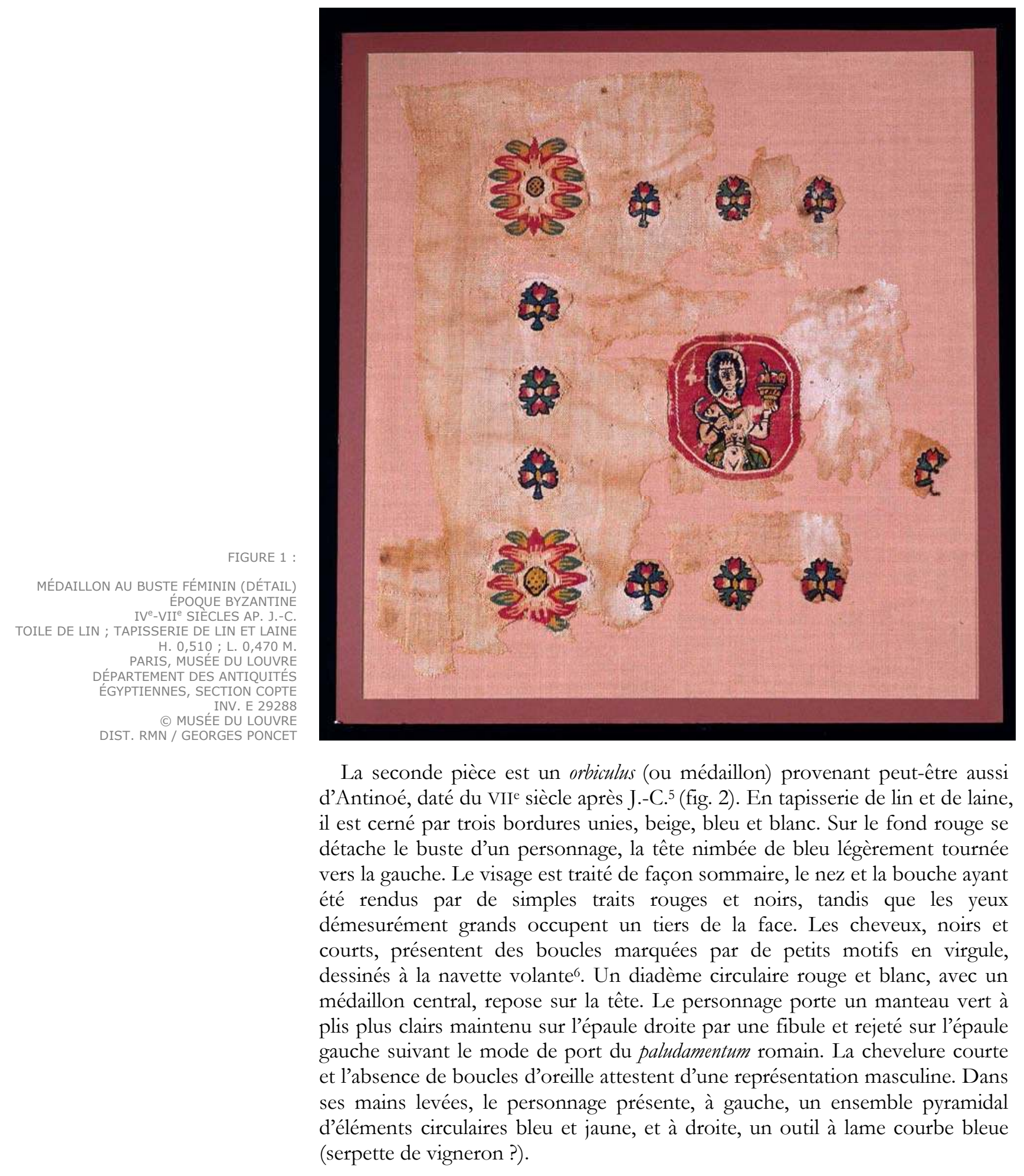

La seconde pièce est un orbiculus (ou médaillon) provenant peut-être aussi d'Antinoé, daté du VII siècle après J.-C. ${ }^{5}$ (fig. 2). En tapisserie de lin et de laine, il est cerné par trois bordures unies, beige, bleu et blanc. Sur le fond rouge se détache le buste d'un personnage, la tête nimbée de bleu légèrement tournée vers la gauche. Le visage est traité de façon sommaire, le nez et la bouche ayant été rendus par de simples traits rouges et noirs, tandis que les yeux démesurément grands occupent un tiers de la face. Les cheveux, noirs et courts, présentent des boucles marquées par de petits motifs en virgule, dessinés à la navette volante ${ }^{6}$. Un diadème circulaire rouge et blanc, avec un médaillon central, repose sur la tête. Le personnage porte un manteau vert à plis plus clairs maintenu sur l'épaule droite par une fibule et rejeté sur l'épaule gauche suivant le mode de port du paludamentum romain. La chevelure courte et l'absence de boucles d'oreille attestent d'une représentation masculine. Dans ses mains levées, le personnage présente, à gauche, un ensemble pyramidal (serpette de vigneron ?).

5. Inv. musée du Louvre E 25346. Pierre du Bourguet, Musée national du Louvre. Catalogue des étoffes coptes, Paris, Réunion des musées nationaux, 1964, D 42, p. 134.

6 . Fil de trame tendu sur l'endroit entre deux points de la pièce, passant par-dessus le fond en tapisserie, et permettant de dessiner de façon plus fine les parties du décor, en même temps que le fond. 


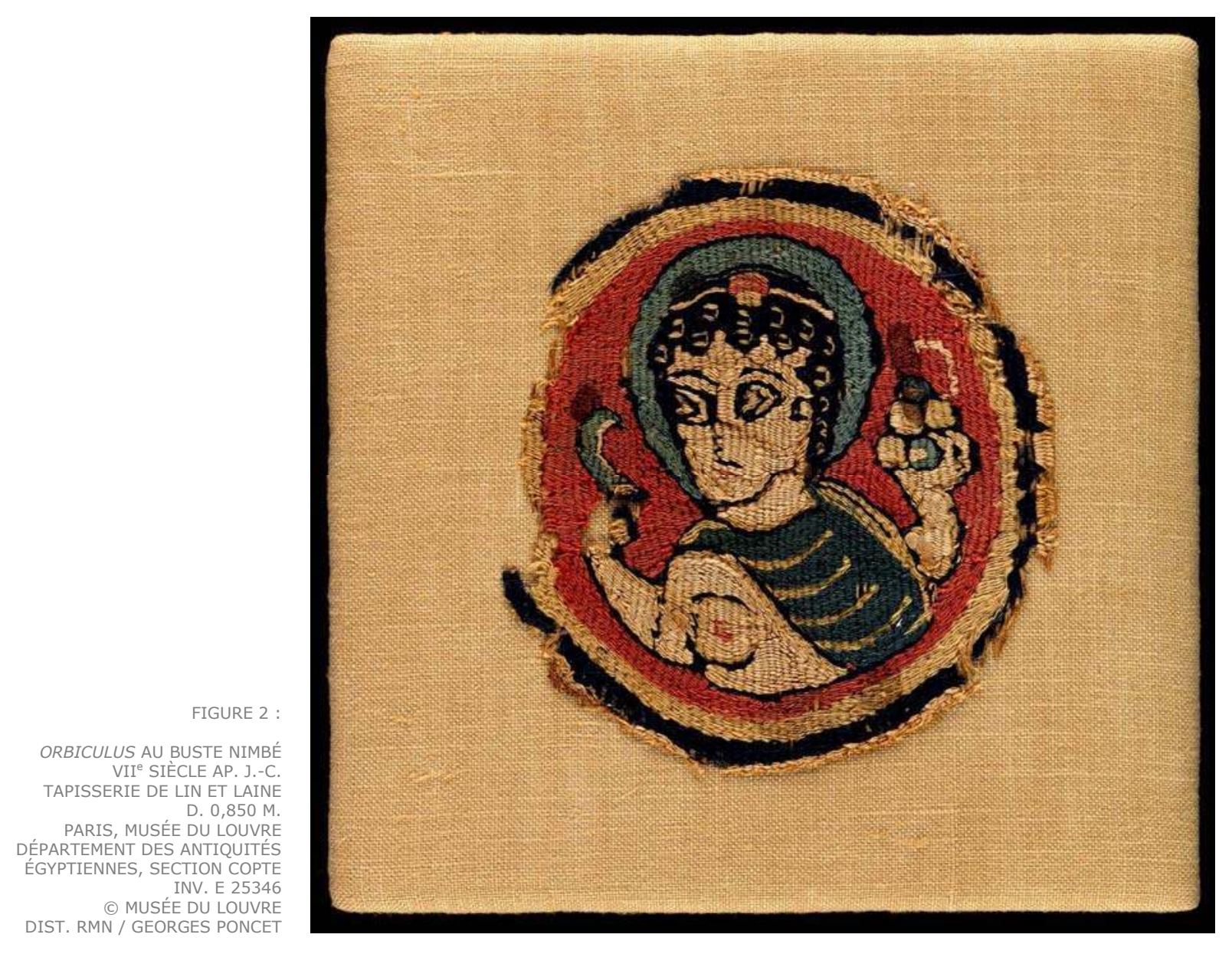

La troisième, une tabula (ou carré) en tapisserie de lin et laine datée du VIII siècle après J.-C.7, est ceinte par une bordure composée de médaillons rouges ornés de « $Z$ » noirs inscrits dans un cadre noir (fig. 3). Le carré central, délimité par une bande rouge puis un filet écru, présente un ovale cerclé d'une bande noire comprise entre deux lignes écru. Un motif ondulé écru vient occuper les écoinçons, tandis qu'au centre du médaillon se détache, sur un fond bleu-vert pâle, le buste d'un personnage. Le traitement du visage est fruste et géométrique, les yeux ayant été rendus par de grands carrés, le nez par une équerre et le menton par un ovale. Deux traits rouges marquent la bouche et une ride frontale. La chevelure, parée d'un diadème à gemmes ocre, écru et vert foncé, est séparée en deux mèches au niveau des tempes pour se terminer en un chignon sommital. Le personnage porte un vêtement flammé de rouge et de rose, présentant sur le torse un plastron richement orné, composé de deux carrés superposés, gemmé d'ocre et de bleu pâle en partie supérieure, et d'un semis de carrés bleu-vert pâle en bas. Malgré les lacunes, la position des bras peut être déterminée par la présence de deux attributs, figurés de part et d'autre de la tête, que notre personnage tenait dans chaque main. Il s'agit à gauche d'une tige noire d'où s'échappe un pétale cordiforme rouge à cœur écru, et à droite, d'un autre élément végétal, encadré par deux tigelles noires disposées en arceau, surmontant un élément recourbé bleu-vert pâle.

7. Déposé au musée de Rennes depuis septembre 1908 (inv. D.08.5.78.7) par le Musée national du Moyen Âge-Thermes et hôtel de Cluny (inv. Cl. 16675). Alexandra Lorquin, Les Tissus coptes au Musée national du Moyen-Âge-Thermes de Cluny, Paris, Réunion des musées nationaux, 1992, pp. 173-174. 


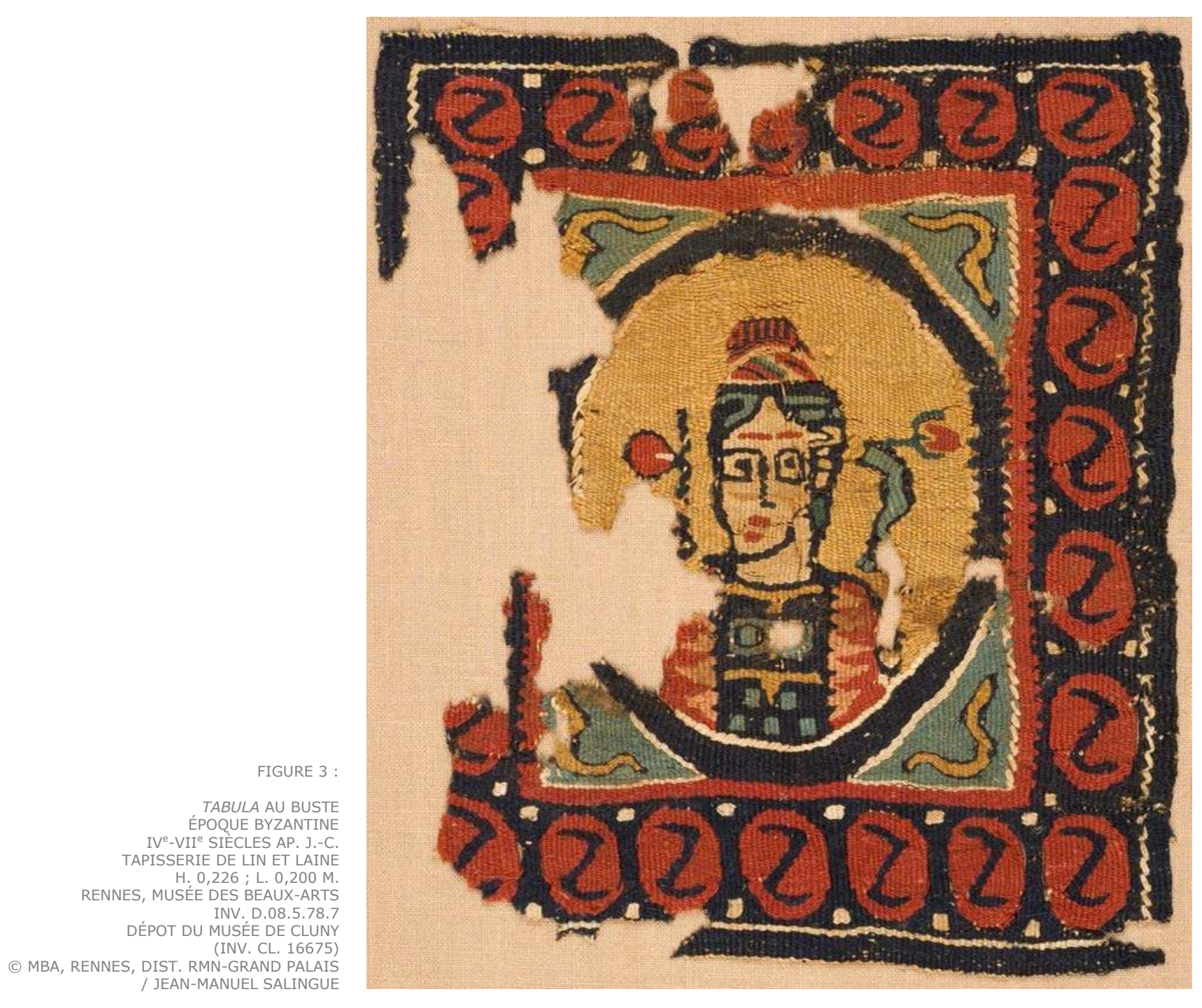

\section{Les clefs de compréhension}

Deux de ces trois textiles (fig. 1 et 2) présentent des figures, la tête ceinte d'un nimbe. Selon Joan Allgrove McDowell ${ }^{8}$, spécialiste des arts textiles d'Orient à l'Université de Cambridge, le nimbe, bien avant d'être repris par les arts chrétiens, constituait un attribut romain fortement répandu durant l'Antiquité tardive, utilisé pour mettre en valeur les personnages illustres, historiques, mythologiques ou allégoriques. Sur les textiles, un facteur d'ordre pratique semble en outre avoir été à l'origine du recours fréquent mais non systématique à ce type de halo, la représentation d'une tête aux cheveux bruns sur un fond sombre posant un problème de lisibilité, ainsi résolu par l'emploi d'une bande plus claire faisant contraste. A contrario, celle-ci perd toute nécessité lorsque le personnage se détache sur un fond clair, comme c'est le cas sur notre troisième textile où le nimbe est absent, malgré un thème iconographique similaire à celui des deux autres pièces.

Ces trois personnages ont été traités de façon géométrique et sommaire, contrairement aux objets qui leur sont associés, dont le volume rendu, par des plages colorées plus claires dans un souci de réalisme et de mise en relief, contraste avec la planéité des visages et des corps. Ainsi mis en avant, ces différents éléments apparaissent comme les clefs de lecture de ces tissus, et 
nous permettent d'envisager une compréhension d'ordre symbolique, et plus précisément allégorique ${ }^{9}$, de ces représentations, constat qui trouve un écho dans la citation de Charles-Nicolas Cochin et Hubert-François Gravelot, deux artistes du XVIII siècle, selon lesquels "l'intelligence de l'allégorie s'acquiert par la connaissance approfondie des attributs et des emblèmes imaginés par les Anciens et que l'usage a consacrés $»^{10}$.

Ces personnages sont figurés les bras levés afin de présenter deux objets, dont l'un, le petit couteau à lame recourbée, est présent sur chacun des trois tissus, et se rapproche des faucilles et serpettes découvertes sur les sites archéologiques de même époque ${ }^{11}$. Ce type d'outils se retrouve en outre sur un support iconographique contemporain de nos textiles, les mosaïques. Celles-ci, conservées en grand nombre dans tout l'Empire romain, en particulier en Afrique du Nord, apportent des témoignages iconographiques importants, repris dans les provinces, où modèles classiques et influences locales se mêlent pour former un art original, marqué par l'identité de chacune ${ }^{12}$. La mosaïque au Triomphe de Neptune de La Chebba ${ }^{13}$ en Tunisie, datée du II siècle après J.-C., présente à chaque angle la représentation féminine en pied d'une des quatre saisons. La figure de l'Été y est représentée nue, suggérant ainsi l'ardeur de la saison, au milieu d'épis, tenant dans la main droite une faucille et dans la gauche une corbeille chargée de blés, selon un traitement iconographique similaire à celui du tissu du Louvre (fig. 1). Quelques éléments diffèrent néanmoins, témoignant d'une adaptation locale des modèles classiques en accord avec le mode de vie et les croyances égyptiennes. Ainsi, les blés débordant de la corbeille de l'Été de la mosaïque se réduisent sur le tissu à un seul épi, jaillissant au-dessus des fruits. L'élément cruciforme surplombant la faucille pourrait représenter l'étoile Sirius. Le lever héliaque de cet astre en juillet coïncidait, selon le calendrier traditionnel égyptien ${ }^{14}$, avec le début de la crue, dont les bienfaits permettaient la germination et la maturation des produits de la terre, présentés par notre personnage.

Sur le deuxième textile (fig. 2), l'outil à lame recourbée est associé à un autre type de fruits, impliquant certainement une fonction et donc une identification du personnage différentes. En effet, ce dernier tient dans la main gauche un ensemble d'éléments circulaires évoquant la forme d'une grappe de raisins, d'où semble s'échapper une vrille de cep traitée en arrondiments ${ }^{15}$. Or, sur la mosaïque des Saisons de la maison du Drinking Contest à Antioche ${ }^{16}$, le putto ailé représentant l'Automne porte sur l'épaule gauche une corbeille pleine de raisins,

9. L'allégorie, du grec allon, « autre chose » et agoreùein, « parler en public », est le fruit d'un procédé mental consistant à présenter un objet codifié à l'esprit afin de lui donner l'idée d'un autre, et plus particulièrement d'un concept spirituel ou abstrait. Institut de France, Dictionnaire de l'Académie française, neuvième édition, Paris, 1, 1992.

10. Hubert-François Gravelot et Charles-Nicolas Cochin, Iconologie par figures ou traité complet des allégories, emblèmes, etc. Ouvrage utile aux artistes, aux amateurs et pouvant servir à l'éducation des jeunes personnes, Paris, s. d. [vers 1789]. André Masson, L'Allégorie, Paris, P.U.F., 1974, p. 6.

11. Dominique Benazeth, Musée du Louvre. Catalogue du Département des Antiquités égyptiennes. L'art du métal au début de l'ère chrétienne, Paris, Réunion des musées nationaux 1992, p. 272.

12. Philippe Bruneau, La Mosaïque antique, Paris, Presse de I'Université de Paris-Sorbonne, 1987, pp. 48-49.

13. Ville côtière du Sahel tunisien spécialisée depuis l'époque romaine dans le commerce de I'huile d'olive, La Chebba compte, parmi les vestiges retrouvés, les villae de riches propriétaires où les mosaïques abondent. David Parrish, Seasons Mosaics of Roman North Africa, Rome, G. Bretschneider, 1984, pp. 431-435.

14. Danielle Bonneau, «Le cycle du Nil : aspect administratif à l'époque gréco-romaine », Bulletin de l'Institut Français d'Archéologie orientale, Le Caire, 120, 1991, pp. 7-19.

15. Procédé de tissage consistant à enrouler d'un tour le fil de trame sur un ou plusieurs fils de chaîne. Nicole Viallet, Principes d'analyse scientifique. Tapisserie. Méthode et vocabulaire, Paris, Inventaire général des monuments et richesses artistiques de le France, 1971, p. 44.

16. Fondée en 300 avant J.-C. sur la frontière turco-syrienne, Antioche est la troisième ville de I'Empire romain après Rome et Alexandrie, et l'un des berceaux du christianisme. La mosaïque, datée du $\mathrm{VI}^{\mathrm{e}}$ siècle après J.-C. et conservée au Virginia Museum of Fine Arts, Richmond, provient d'une villa romaine à laquelle elle a donné son nom. Fatih Cimok (ed.), A Corpus. Antioch Mosaïcs, Istanbul, 2000, pp.139-143. 
probablement coupés grâce à la serpette tenue dans sa main droite. De la même façon, sur la tenture de Dionysos conservée à l'Abegg-Stiftung (Berne, Suisse $)^{17}$, l'un des personnages du cortège divin présente ce même outil, ce qui tendrait à en faire un attribut représentatif des activités automnales, saison dont Dionysos est le dieu tutélaire. L'association de cette serpette et de la grappe de raisin sur notre textile permettrait donc d'affirmer qu'il s'agit d'une représentation allégorique de l'Automne.

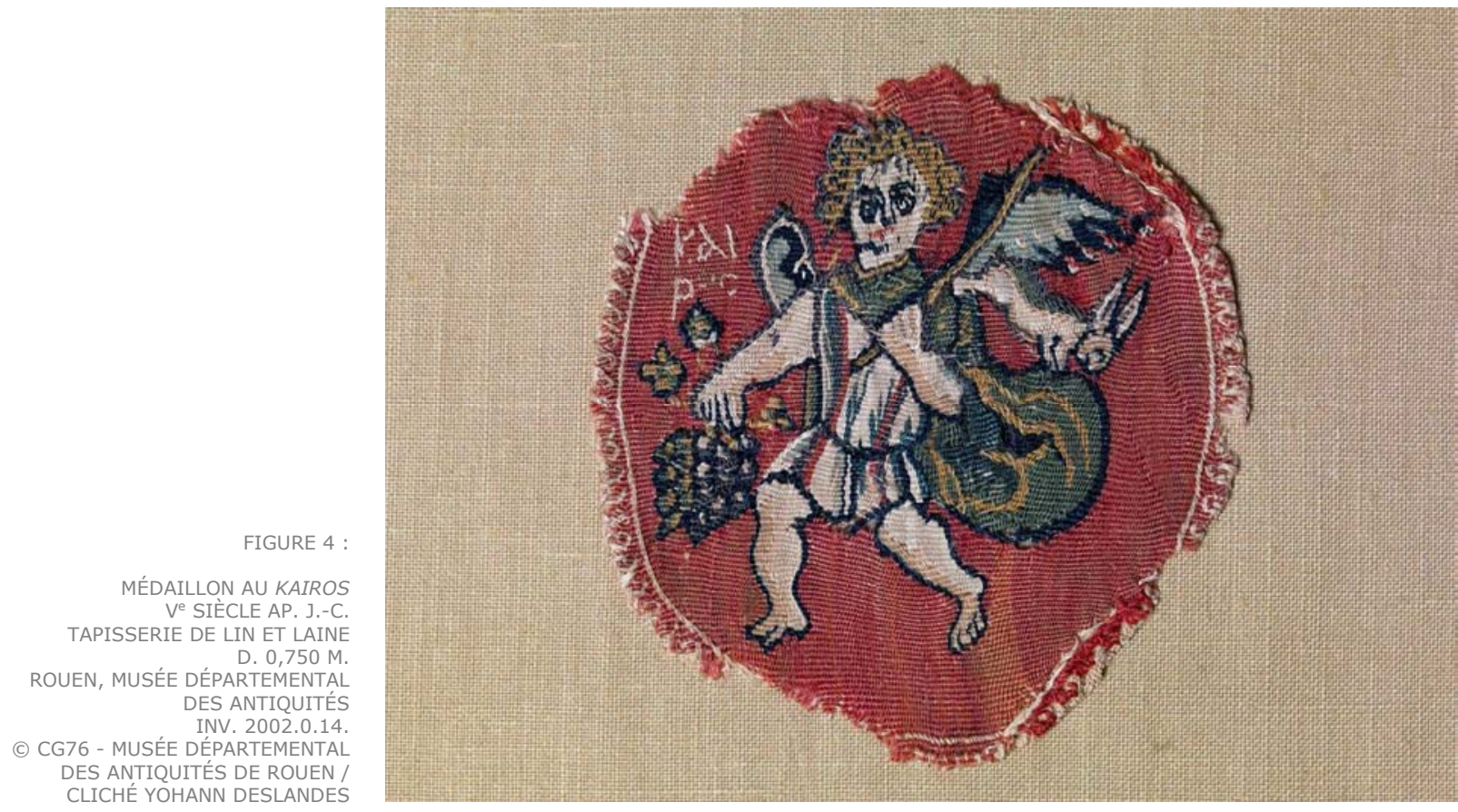

Le même outil se retrouve enfin dans la main du troisième personnage (fig. 3), associé, dans l'autre main, à un bâton sec, symbolisant le dénuement caractéristique de l'Hiver, selon un schéma proche de celui d'un orbiculus conservé au musée de Rouen ${ }^{18}$ (fig. 4). Sur ce dernier, à fond rouge et frise de postes, un putto ailé en tunique courte et manteau vert tient dans la main gauche, un bâton au bout duquel est suspendu un lièvre, renvoyant aux chasses hivernales, et dans la droite, une grappe de raisin, que notre tisserand aurait donc remplacée, dans la main gauche de notre personnage, par une serpette (fig. 3). Sur l'orbiculus de Rouen, une inscription grecque nous donne l'identité


opportun » ou l'« Occasion».

Maximilien Durand a récemment démontré que cet orbiculus appartenait à une tunique ornée de clavi et de deux autres médaillons inscrits conservés au musée du Louvre ${ }^{19}$, aux représentations allégoriques du Printemps ( $\varepsilon \propto \varrho \mathrm{v} \eta$ )

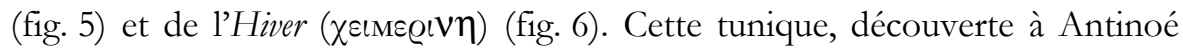
par Albert Gayet pendant les fouilles de 1900-1901, est présentée dans sa Notice de $1901^{20}$ comme une «tunique de dessous avec médaillons sur le bas, donnant les figures auréolées du printemps et de l'hiver». Ces deux saisons, nommées, sont en outre reconnaissables à leurs attributs caractéristiques.

17. Inv. 3100a. Antoine De Moor, Cäcilia Fluck, Clothing the House. Furnishing Textiles of the $1^{\text {st }}$ Millennium AD from Egypt and neighbouring countries, Tielt, 2009, pp. 146-147.

18. Inv. 2002.0.14. Cat. d'exp., Égypte, la trame de I'histoire. Textiles pharaoniques, coptes et islamiques, Rouen-Roanne-Paris, 2002-2004, $\mathrm{n}^{\circ} 108$.

19. Inv. AF 5506. Maximilien Durand, op. cit. note 18, $\mathrm{n}^{\circ} 107$.

20. Albert Gayet, Notice relative aux objets recueillis à Antinoé pendant les fouilles exécutées en 1900-1901 et exposés au musée Guimet du 15 juin au 31 juillet 1901, Paris, E. Leroux, 1901 , p. 27. 
FIGURE 5 (A GAUCHE) :

MÉDAILLON A L'ALLÉGORIE DU PRINTEM Ve SIECLE AP. J.-C. TAPISSERIE DE LIN ET LAINE

PARIS, MUSÉE D. 0,800 M. DÉPARTEMENT DES ANTIQUITÉS ÉGYPTIENNES, SECTION COPTE INV. AF 5506 C RMN-GRAND PALAIS (MUSÉE DU LOUVRE)

/ HERVÉ LEWANDOWSKI

FIGURE 6 (A DROITE) :

MÉDAILLON A L'ALLÉGORIE DE L'HIVER $V^{e}$ SIECLE AP. J.-C TAPISSERIE DE LIN ET LAINE

PARIS, MUSÉE D. 0,800 M. DÉPARTEMENT DES ANTIQUITÉS EGYPTIENNES, SECTION COPTE INV. AF 5506

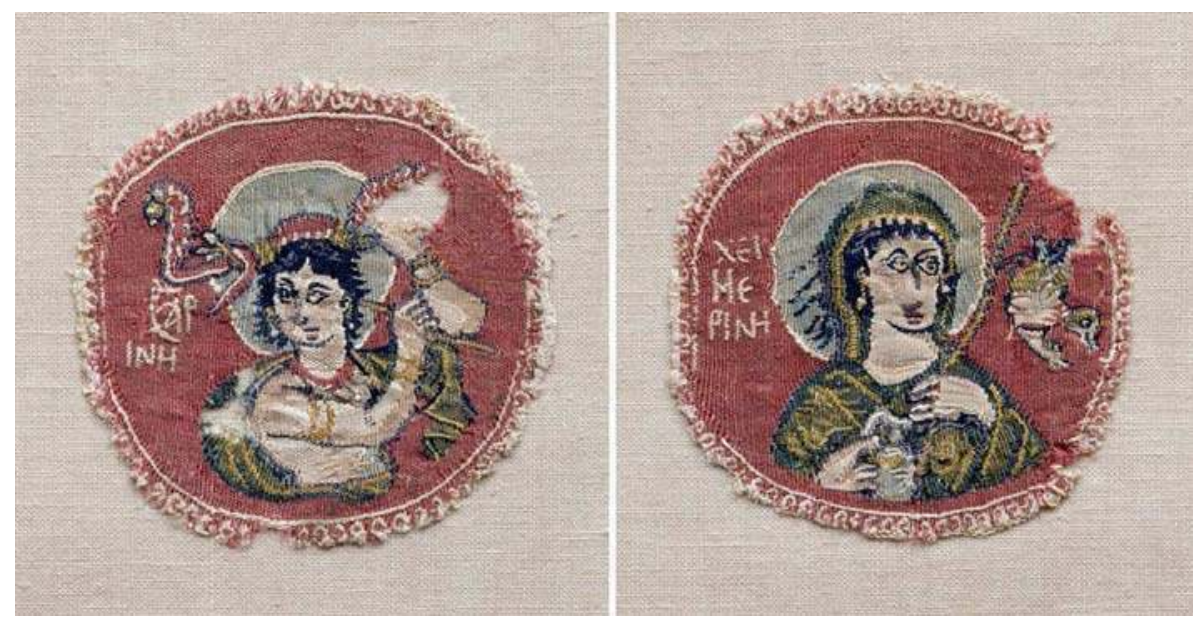

Sur le premier orbiculus (fig. 5), une femme au nimbe bleu clair, est représentée nue, un simple voile vert venant lui couvrir le ventre, passant derrière l'épaule droite et retombant en plis flottant sur l'épaule gauche. Les deux bras levés, ainsi que les poignets, sont parés de doubles bracelets dorés, tandis qu'un collier ou un ruban rouge orne la base du cou. Sur sa tête repose un bandeau ou un voile rouge maintenu par deux fleurons jaunes et verts. La main droite serre un objet lacunaire, dont la forme et le traitement iconographique laissent penser à une couronne de fleurs. Le bras gauche tient un bâton orné de deux gemmes, passant derrière la tête et à l'extrémité duquel est attachée une bandelette de tissu rouge, bleu et blanc ${ }^{21}$. Il s'agit ici d'un thyrse, attribut traditionnel du dieu Dionysos, dont l'association à la figure du Printemps constituerait une originalité qui se retrouverait exclusivement sur les mosaïques d'Afrique du Nord, afin de faire allusion aux fêtes dionysiaques qui avaient lieu au mois de mars ${ }^{22}$. L'orbiculus du Printemps appartenant aux collections du musée du Louvre semble ainsi s'inscrire dans cette tradition iconographique propre aux provinces romaines d'Afrique du Nord. Toutefois, l'attitude générale de notre personnage, dont le buste se tord vers la droite, reste sans équivalent à notre connaissance et semble donc constituer une particularité égyptienne.

Le second orbiculus (fig. 6) présente également une femme nimbée de bleu clair et emmitouflée dans un manteau vert et jaune, la tête recouverte d'un capuchon. À sa droite se trouve l'inscription grecque qui la nomme, $\chi \varepsilon \iota \mathrm{e} \varrho \iota \eta \eta$, l'Hiver. Conformément aux représentations de cette saison, elle porte dans la main droite un rhyton, symbolisant l'humidité de cette période de l'année et les orgies saturnales, et dans la gauche, un bâton au bout duquel sont suspendus deux canards morts, comme évocation des chasses hivernales.

L'observation de ces deux orbiculi témoigne ainsi, pour cette tunique, d'un programme iconographique articulé autour du thème des Saisons. Cependant, le terme grec associé au putto de Rouen (fig. 4) appartenant à cette même tunique

21. Pour une représentation identique d'un thyrse, voir Adolphe-Joseph Reinach, « Thyrse », dans Charles Daremberg, Edmond Saglio (dir.), Dictionnaire des Antiquités grecques et romaines, vol. 9, Saint-Germain-lès-Corbeil, 1877-1913, fig. 6923 et pp. 291-293. La présence du ruban est à imputer à la présence originelle sur les hampes des thyrses de touffes de feuilles, que maintenaient ces bandelettes. A partir du IV siècle avant J.-C., presque tous les thyrses se parent de bandelettes, même si ces touffes végétales font défaut, afin d'en souligner l'aspect décoratif et le caractère sacré. Ce type de représentation, véhiculé dans tout I'Empire romain, a été récupéré en Égypte au cours des $\mathrm{IV}^{\mathrm{e}}-\mathrm{III}^{\mathrm{e}}$ siècles avant notre ère, comme en atteste la pompè de Ptolémée II, où on voit Nysa représentée tenant un thyrse entouré de bandelettes (voir Callixène, ap. Athens 198). Enfin, une élégie anonyme sur la mort de Mécène nous informe sur le fait que la décoration du thyrse peut aussi se parer d'or et de pierreries, comme c'est ici le cas (voir Elegia in obitum Maecenatis, V. 63). Symbole de prospérité et de fertilité, le thyrse était porté par tous les fidèles de Dionysos durant les fêtes orgiaques.

22. Cat. d'exp., Égyptes... L'Égyptien et le Copte, Musée archéologique Henri Prades, Lattes, $1999, n^{\circ} 83$. 
ne désigne pas, comme nous aurions pu l'attendre, l'Automne ou l'Été, mais un des génies grecs des moments de l'année, les Kairoi, amours ailés accompagnant les saisons durant l'Antiquité tardive, dont ils symbolisent les fruits et les richesses. Selon Maximilien Durand, il s'agirait alors d'une représentation de l'Automne, dont le traitement serait «contaminé » par les représentations des Kairoi.

Néanmoins, le sens de chaîne ${ }^{23}$ de cette pièce n'est pas identique à celui des deux orbiculi aux Saisons du Louvre : le premier est horizontal, tandis que les deux autres sont verticaux, suivant le sens de lecture. Les tuniques étaient tissées en une pièce, en commençant par une manche pour terminer par l'autre, les décors en tapisserie répartis symétriquement sur le vêtement étant exécutés en même temps sur le métier. Dès lors, une telle différence des sens de chaîne impliquerait une destination ornementale différente pour les deux Saisons (épaules), et pour le Kairos (genoux), laissant ainsi supposer l'existence originelle de trois autres Kairö̈, placés aux genoux (fig. 7).

FIGURE 7 :

HYPOTHÈSE DE RÉPARTITION DES DÉCORS SUR LA TUNIQUE DÉCOUVERTE PAR ALBERT GAYET À ANTINOÉ : DÉCORS TISSÉS EN MÊME TEMPS QUE LE FOND CROQUIS DE L'AUTEUR (C) AMANDINE MÉRAT

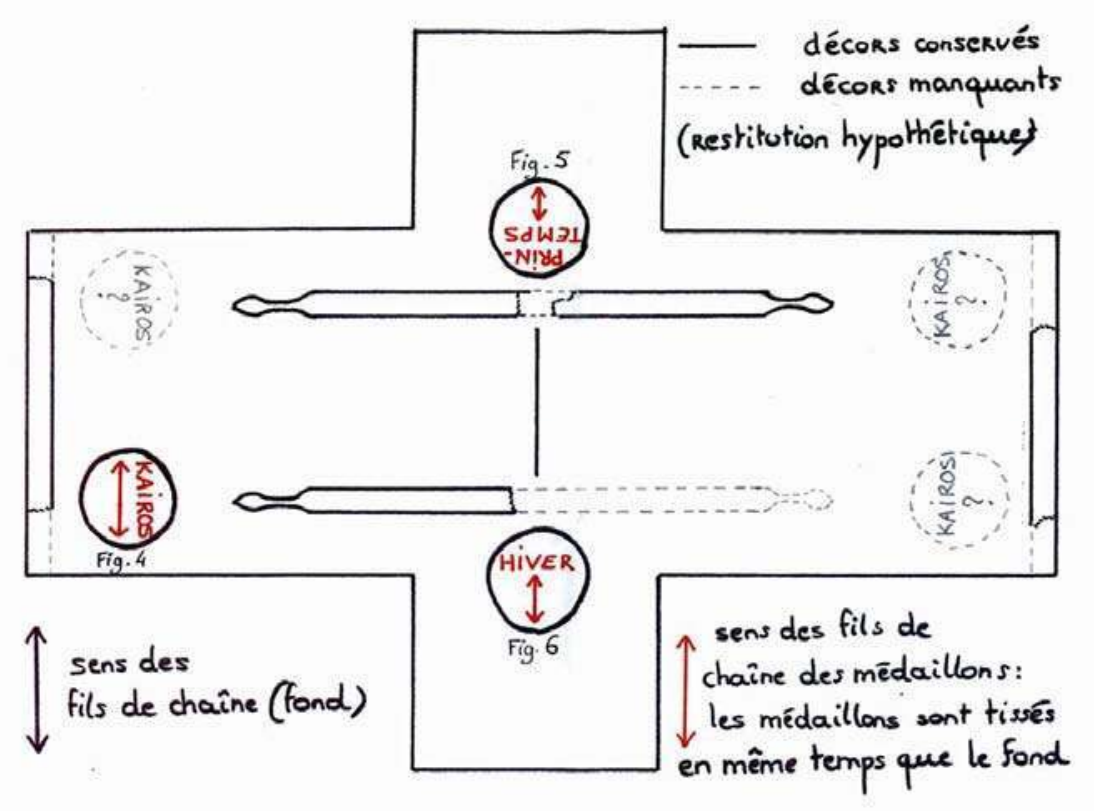

Or, dans sa Notice, Albert Gayet indique que les médaillons aux Saisons étaient placés «en bas » du vêtement, à savoir aux genoux. Cette hypothèse impliquerait que les divers décors en tapisserie de cette tunique n'aient pas été tissés en même temps que le fond du vêtement, mais rapportés par couture durant l'Antiquité. Tisser un vêtement orné de tapisserie représentait en effet un travail fastidieux et précieux, aussi les Égyptiens récupéraient-ils fréquemment les ornements en tapisserie des pièces usagées pour en parer de nouvelles. Le Printemps et l'Hiver ayant été placés aux genoux, ceci entraînerait, suivant le principe de symétrie, l'existence de deux autres médaillons au revers, portant les représentations nommées de l'Automne et de l'Été. Le Kairos de Rouen aurait alors orné une des épaules, entre les représentations de l'Automne et de l'Hiver dont il porte les attributs, position qui justifierait ainsi l'inscription xaı@os, conforme à l'identité du personnage. Un deuxième Kairos, portant peut-être les attributs de l'Été et du Printemps, serait alors placé en symétrie sur l'autre épaule (fig. 8). La répartition des décors de cette tunique suivrait ainsi

23. Fils généralement écrus tendus parallèlement sur le métier, entre lesquels sont passés, perpendiculairement et au moyen d'une navette, les fils de trame qui confectionnent le décor et recouvrent complètement la chaîne une fois le tissage terminé. 
un programme iconographique cohérent, visant à reproduire le cycle des Saisons et des « Moments opportuns » de l'année.

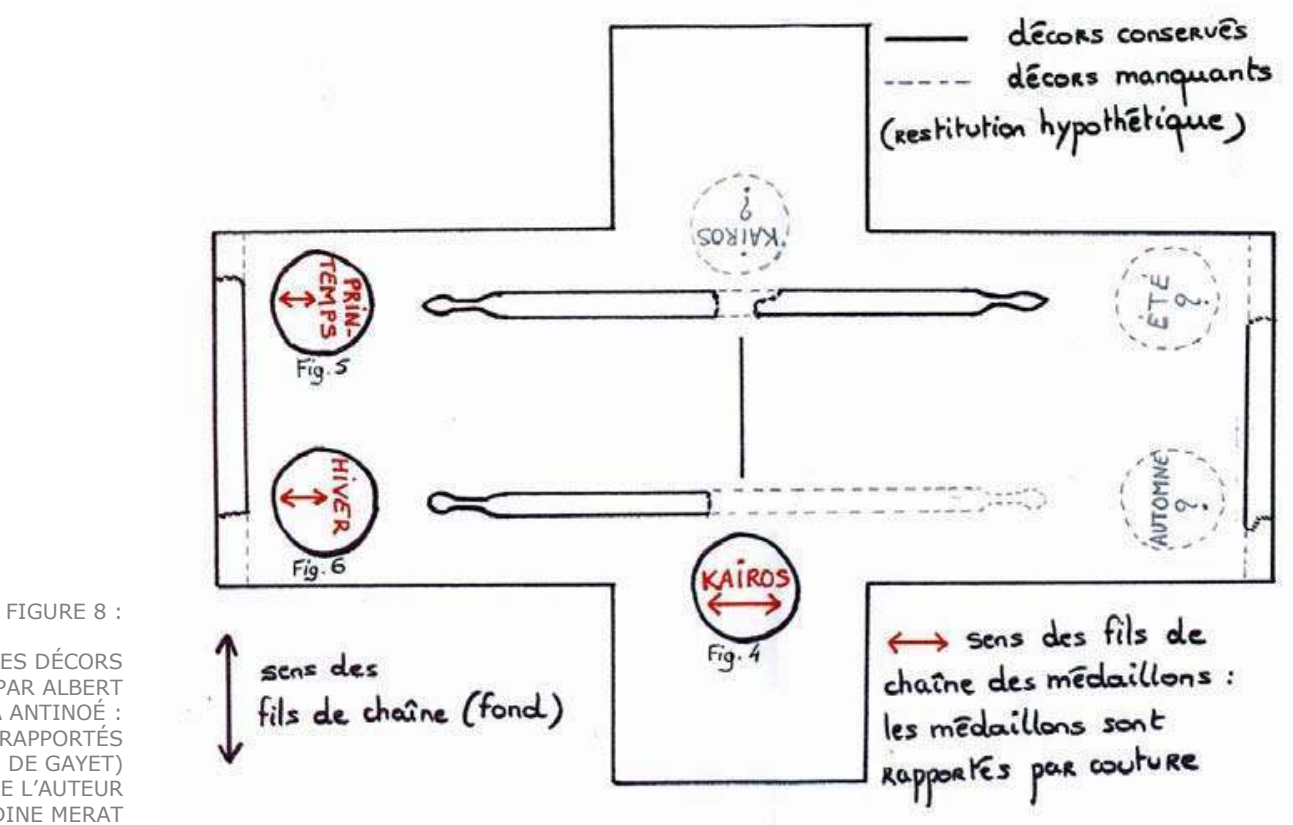

Bien que figuré en buste, le traitement iconographique du personnage de la tabula (fig. 3), présentant également les attributs caractéristiques de l'Automne et de l'Hiver, pourrait s'apparenter à celui du Kairos de Rouen. Par comparaison, l'analyse précédente permet de nous éclairer sur l'identité de ce personnage privé d'inscription, qui pourrait donc représenter une allégorie masculine de l'Automne influencée par l'iconographie des Kairoi, ou encore l'un de ces génies lui-même, selon nos propres observations. L'existence d'une tabula conservée au musée des Arts Décoratifs de Paris ${ }^{24}$, au personnage et au traitement stylistique identiques à celui de la tabula de Rennes pourrait alors confirmer cette seconde hypothèse.

\section{Les allégories des Saisons}

Durant les premiers siècles de notre ère, des témoignages littéraires ${ }^{25}$ et artistiques attestent de la récupération par les Égyptiens du thème iconographique romain des quatre Saisons ${ }^{26}$, et ceci malgré un calendrier traditionnellement basé sur le cycle de la crue du Nil, divisant l'année en trois saisons ${ }^{27}$.

Représentations figurées découlant d'un concept abstrait lié à la perception que l'homme a du monde qui l'entoure, les allégories des Saisons apparaissent dans la tradition mythologique orale grecque, avant que leurs légendes ne soient " couchées sur le papier » dans les récits antiques dès le VIII e siècle avant J.-C. Leur symbolisme y est alors décrit : ce sont elles qui président au cycle permanent du Temps, rapportant immuablement aux hommes les fruits et les richesses qui garantissent bonheur et prospérité. Par extension, elles en viennent à recouper des notions de naissance, croissance, mort et renaissance,

24. Tabula provenant des fouilles d'Antinoé, conservée dans l'Album du Musée des Arts Décoratifs de Paris, p. 34.

25. Nonnos de Panopolis, Les Dionysiaques, traduction de F. Vian, Paris, V, 1995, chant XI.

26. Kamila Kolodziejczyk, « Fragments d'enduits peints des fouilles polonaises à Kom el Dikka (Alexandrie) », Études et Travaux. Travaux du Centre d'archéologie méditerranéenne de I'Académie des sciences polonaises, Varsovie, 1990, p. 193-202.

27. Danielle Bonneau, op. cit. note 14 . 
expliquant certainement le succès qu'elles connurent dans les arts grécoromains, et leur association à des contextes funéraires païens et chrétiens.

Ce phénomène pourrait en outre s'expliquer par une croyance populaire commune à l'ensemble des productions imagées de cette époque, rapportée par Achille Tatius, écrivain grec des $\mathrm{II}^{\mathrm{e}}-\mathrm{III} \mathrm{I}^{\mathrm{e}}$ siècles après J.-C., originaire d'Alexandrie : «Les interprètes de présages conseillent de faire attention au sujet des tableaux que l'on peut rencontrer lorsqu'on sort pour telle affaire: ils affirment que l'issue de celle-ci sera semblable à celle contée par le tableau. ${ }^{28}$ »

Ainsi, si les scènes représentées sur les supports artistiques sont associées à des idées de prospérité et de bonheur, le simple fait de les observer permettrait que leurs bienfaits s'accomplissent en faveur de qui les contemple ou les produit. En Afrique du Nord, le motif des Saisons est un thème iconographique récurrent sur les mosaïques, comme l'attestent les quatre-vingt-deux exemples, destinés tant aux édifices publics qu'aux maisons privées, recensés par David Parrish pour la seule Afrique proconsulaire ${ }^{29}$. Or, selon Philippe Bruneau ${ }^{30}$, la mosaïque découlerait directement des tapis ornant les sols des villae, dont elles seraient le substitut moins fragile. Les liens entre textile et mosaïque se révèlent donc étroits, et les parentés iconographiques d'un support à l'autre, nombreuses, comme en témoignent notamment les bordures géométriques ou végétales communes aux deux arts.

Au vu des formes et dimensions des textiles étudiés, il semblerait que nous soyons en présence de trois types de pièces distincts: une pièce d'ameublement de type coussin pour notre premier textile (fig. 1), une tabula ayant pu orner une tenture ou un châle pour le textile de Rennes (fig. 3), des médaillons décoratifs d'habillement pour les orbiculi de Rouen (fig. 4) et ceux du Louvre (fig. 2, 5 et 6). La pièce de Rouen (fig. 4) et celles du Louvre (fig. 5 et 6), qui appartiennent à une même tunique, constituent un témoignage rare de programme iconographique complexe conservé sur un textile d'habillement, et attestent avec certitude de la connaissance et de la récupération par les Égyptiens du thème des Saisons et de sa signification.

En gardant à l'esprit que l'Égypte des premiers siècles de notre ère s'inscrit dans la lignée de la tradition artistique et culturelle de l'Empire romain, selon laquelle la simple représentation d'une allégorie permettait d'attirer protection et prospérité à qui la contemple ou la porte sur soi, il semble donc envisageable que cette même symbolique ait été récupérée par les Égyptiens pour venir orner leurs tissus d'ameublement ou d'habillement. Ces derniers se placeraient donc encore, à l'époque où le christianisme apparait puis se répand en Égypte, sous la protection d'allégories païennes représentées sous des traits féminins ou masculins, parfois mises en valeur par la présence de nimbes selon la tradition romaine, et dotées d'attributs identitaires.

28. Achille Tatius, Leucippé et Clitophon, traduction de J.-P. Garnaud, Paris, V, 1991, vers 4. 29. Province romaine conquise en 146 avant J.-C., correspondant au territoire de Carthage (actuelle Tunisie) et à la côte occidentale libyenne. David Parrish, op. cit. note 13.

30. Philippe Bruneau, op. cit. note 12 . 


\section{Pour citer cet article}

\section{Référence électronique}

Amandine Mérat, " Des allégories des Saisons sur les tissus coptes », Cahiers de l'École du Louvre. Recherches en histoire de l'art, histoire des civilisations, archéologie, anthropologie et muséologie [en ligne] $\mathrm{n}^{\circ} 2$, mars 2013, mis en ligne le 15 mars 2013.

http://www.ecoledulouvre.fr/cahiers-de-l-ecole-du-louvre/numero2mars2013/Merat.pdf

\section{(c) creative \\ (B.) $\ominus$ (웅}

\section{L'auteur}

Après un $1^{\text {er }}$ cycle à l'École du Louvre durant lequel elle a suivi les enseignements d'histoire de l'art, archéologie et épigraphie égyptiennes, Amandine Mérat s'est spécialisée au cours de son année de Muséologie dans la coptologie et l'étude des textiles archéologiques. Elle a réalisé en $2^{\text {de }}$ année de $2^{\mathrm{e}}$ cycle (master 2), un mémoire de recherche en histoire de l'art appliqué aux collections consacré à l'étude des allégories de la Terre et des Saisons sur les textiles égyptiens d'époque byzantine, dont est tiré le présent article. Allocataire de recherche de 3e cycle de l'École du Louvre pour l'année 20122013, elle prépare depuis la fin 2010 une thèse portant sur « La broderie dans l'Égypte antique, de l'époque pharaonique à l'époque arabe. Étude technique et iconographique. Influences, échanges et diffusion au sein $\mathrm{du}$ bassin méditerranéen et du Proche-Orient », sous la direction de Roberta Cortopassi, conservateur au Centre de Recherche et de Restauration des Musées de France - filière Arts Décoratifs. 